\title{
ARTICLE
}

\section{Neuromorphic device architectures with global connectivity through electrolyte gating}

\author{
Paschalis Gkoupidenis ${ }^{1}$, Dimitrios A. Koutsouras ${ }^{1} \&$ George G. Malliaras ${ }^{1}$
}

Information processing in the brain takes place in a network of neurons that are connected with each other by an immense number of synapses. At the same time, neurons are immersed in a common electrochemical environment, and global parameters such as concentrations of various hormones regulate the overall network function. This computational paradigm of global regulation, also known as homeoplasticity, has important implications in the overall behaviour of large neural ensembles and is barely addressed in neuromorphic device architectures. Here, we demonstrate the global control of an array of organic devices based on poly(3,4ethylenedioxythiophene):poly(styrene sulf) that are immersed in an electrolyte, a behaviour that resembles homeoplasticity phenomena of the neural environment. We use this effect to produce behaviour that is reminiscent of the coupling between local activity and global oscillations in the biological neural networks. We further show that the electrolyte establishes complex connections between individual devices, and leverage these connections to implement coincidence detection. These results demonstrate that electrolyte gating offers significant advantages for the realization of networks of neuromorphic devices of higher complexity and with minimal hardwired connectivity.

\footnotetext{
${ }^{1}$ Department of Bioelectronics, Ecole Nationale Supérieure des Mines, CMP-EMSE, MOC, Gardanne 13541, France. Correspondence and requests for materials should be addressed to G.G.M. (email: malliaras@emse.fr).
} 
T he main driving force for new developments in contemporary electronics is the evolution from increasing density to increasing functionality. It is now well recognized that electronic circuits based on traditional von Neumann architectures are not well-adapted to capture the real world information processing capability of biological nervous systems ${ }^{1}$. The main reason of this limitation is the so-called von Neumann bottleneck, due to the physical separation of computational and memory units ${ }^{2}$. Overcoming the von Neumann bottleneck can be achieved through the use of computational concepts borrowed from biology. Neuromorphic engineering, established in the late 1980s through the work of C. Mead ${ }^{3}$, involves mimicking the neuronal architectures present in the nervous system with silicon-based circuits. A recent breakthrough in the field is the development of neuro-inspired, multi-core chips with traditional CMOS (Complementary Metal/ Oxide/Semiconductor) technology ${ }^{4}$. At the same time, a host of new devices with intrinsic neuromorphic behaviour gave birth to promising new computational paradigms that go beyond the traditional von Neumann architecture ${ }^{5}$. Several solid-state technologies including memristive, phase-change, spintronic and ferroelectric devices have been used in hardware-based implementations of the basic functional building blocks of neural processing and of the various forms of neuroplasticity ${ }^{6-9}$.

A recent trend in neuromorphic engineering involves the use of devices based on organic electronic materials ${ }^{\mathrm{P}-16}$. This is largely motivated by the attractive characteristics organic devices offer in interfacing electronics with biology ${ }^{17}$. Indeed, the emerging field of organic bioelectronics is making available devices with exceptional performance for a variety of applications including neural interfaces, biosensors, and drug delivery, as well as new concepts such as chemical-based logic, and electronic plants ${ }^{18-22}$. A particularly popular example is the organic electrochemical transistor (OECT), a device that employs a channel made from an organic mixed electronic/ionic conductor, such as the conducting polymer poly(3,4ethylenedioxythiophene):poly(styrene sulf) (PEDOT:PSS). The electronic conductivity of the channel is modulated by ions injected from an electrolyte when an appropriate gate voltage is applied 23,24 . This mechanism of operation facilitates the strong coupling between electronic and ionic charge carriers and enables efficient communication between electronics and biological systems. It has also been used to demonstrate a variety of neuromorphic functions including short-, long-term synaptic plasticity functions and orientation selectivity ${ }^{10-12,25}$.

In the majority of neuromorphic circuits, each device is connected to others through a predefined physical wire network, a design that severely limits the number of connections that can be established between different devices ${ }^{26,27}$. On the other hand, biological information processing involves neural networks of immense connectivity 28 . The human brain for example consists of approximately $10^{11}$ neurons communicating with each other by an interwoven network of almost $10^{14}$ synapses, and each neuron might be connected to up to $10^{4}$ other neurons through these synapses. Synaptic weight expresses the connectivity degree between the neurons through the synapses and typically in computational neuroscience it can be defined as a scalar number between 0 and 1 ( $0-$ no connectivity from neuron to neuron, $1-$ maximum connectivity from neuron to neuron). The structure and the function of this complex network develops over time, resulting thus in memory and learning ${ }^{28}$. On top of all these, global mechanisms that modulate neural processing (that is, homeoplasticity) play a key role in brain function. Ionic concentrations, concentration of various hormones and temperature are some of the parameters that provide global regulation of synaptic strength ${ }^{29,30}$. This homeostatic or global regulation of synaptic strengths is regarded as a normalization mechanism that is necessary for the stabilization of correlationbased rules such as Hebbian plasticity ${ }^{29,31}$. As positive feedback rules tend to destabilize the behaviour of large neural networks, homeostatic phenomena force globally specific constraints in synaptic strengths ${ }^{31-34}$

In this work, we demonstrate the concept of global regulation in neuromorphic device architectures through an electrolyte, a behaviour analogous to homeostasis in the brain (Fig. 1a). We use an array of two-terminal devices immersed in an electrolyte, where each device consists of a PEDOT:PSS channel that serves as a hard connection between an input and an output wire. We show that the weights of these hard connections are modulated globally by the voltage applied on the electrolyte and by its ion concentration, in the way that is analogous to homeoplasticity. We take advantage of this effect to demonstrate synchronization of I/O transmission and global clock behaviour, reminiscent of the coupling between individual neuron activity and global oscillations in biological networks. We further show that the electrolyte establishes complex soft connections (that is, without physical wiring) between individual devices. We leverage these connections to implement coincidence detection, a function used in the visual and auditory systems of the brain, in devices with minimal hard connectivity. These results demonstrate that electrolyte connections offer significant advantages for the realization of networks of neuromorphic devices of higher complexity.

\section{Results}

The concept of global gating. The device architecture is depicted in Fig. 1a to consist of a $4 \times 4$ grid of two-terminal devices in a square configuration, where indexes $i=1-4$ and $j=1-4$ correspond to device number on the grid. As will be presented below, various physical and soft connections can be established between the terminals of the devices and through the electrolyte (Fig. 1b). The fabrication process is described in the experimental section. Briefly, each device consists of a lithographically defined PEDOT:PSS channel that connects two Au wires deposited on a glass substrate. The channels have nominal dimensions of $50 \mu \mathrm{m} \times 50 \mu \mathrm{m}$ (length $\times$ width, $L \times W$ ) and are placed at a centre-to-centre device distance $d=5 \mathrm{~mm}$. The Au wires are covered by an insulating film, therefore only the PEDOT:PSS channels are exposed to a common $100 \mathrm{mM}$ (unless otherwise stated) $\mathrm{NaCl}$ electrolyte in which a $\mathrm{Ag} / \mathrm{AgCl}$ gate electrode is immersed. For each device, one of the Au wires is used as a local input $\left(I_{i}\right)$ and a voltage is applied as the input signal. The other terminal is connected to the ground through an electrometer, and the measured current is defined as the output $\left(O_{i}\right)$. Each device can also be addressed by the global input $(G)$ when a voltage is applied to the gate electrode.

Figure $1 \mathrm{c}-\mathrm{f}$ show how the global input can be used to modulate individual devices. The protocol for the measurements is displayed in Fig. 1c and consists of applying voltage pulses at the local input $I_{i}$, while applying various DC voltages at the global input $G$. The output $O_{i}$ of the device at coordinates $(1,1)$ is shown in Fig. 1d to be suppressed as the global input increases. This can be described as in Fig. 1e, by considering that the connection weight $w_{i i}$ (defined as $O_{i} / O_{\mathrm{MAX}, i}$, where $O_{\mathrm{MAX}, i}$ is the output that corresponds to a global input of $0 \mathrm{~V}$ ) is gradually suppressed by the action of the global input and becomes practically zero for large positive values and for an electrolyte concentration of $100 \mathrm{mM}$. It should be mentioned that the term connection weight is used here instead of the classic definition of synaptic weight, because it has volatile nature and is a metric of how the physical connection can be modulated by using a global gate electrode. 


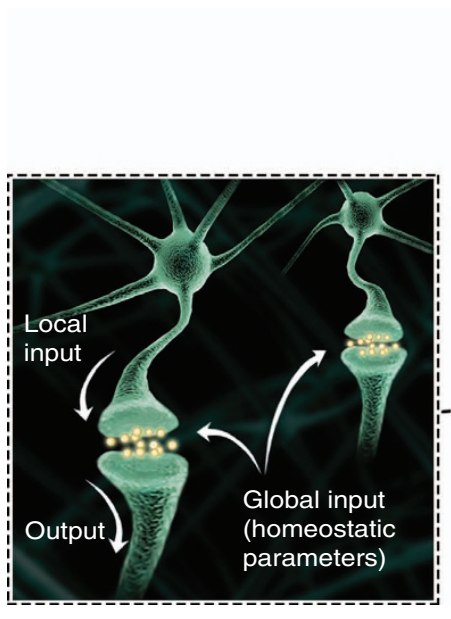

C

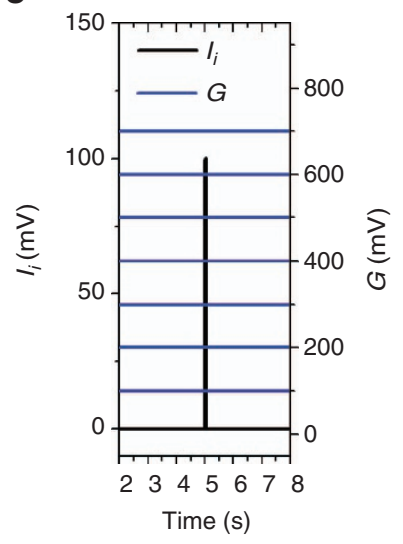

f

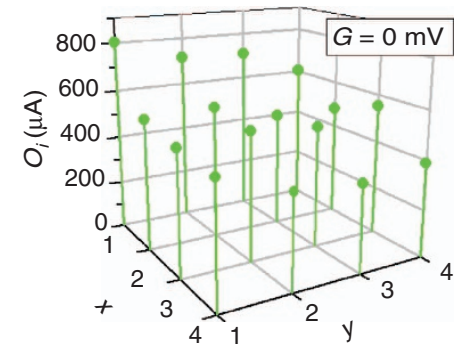

d a
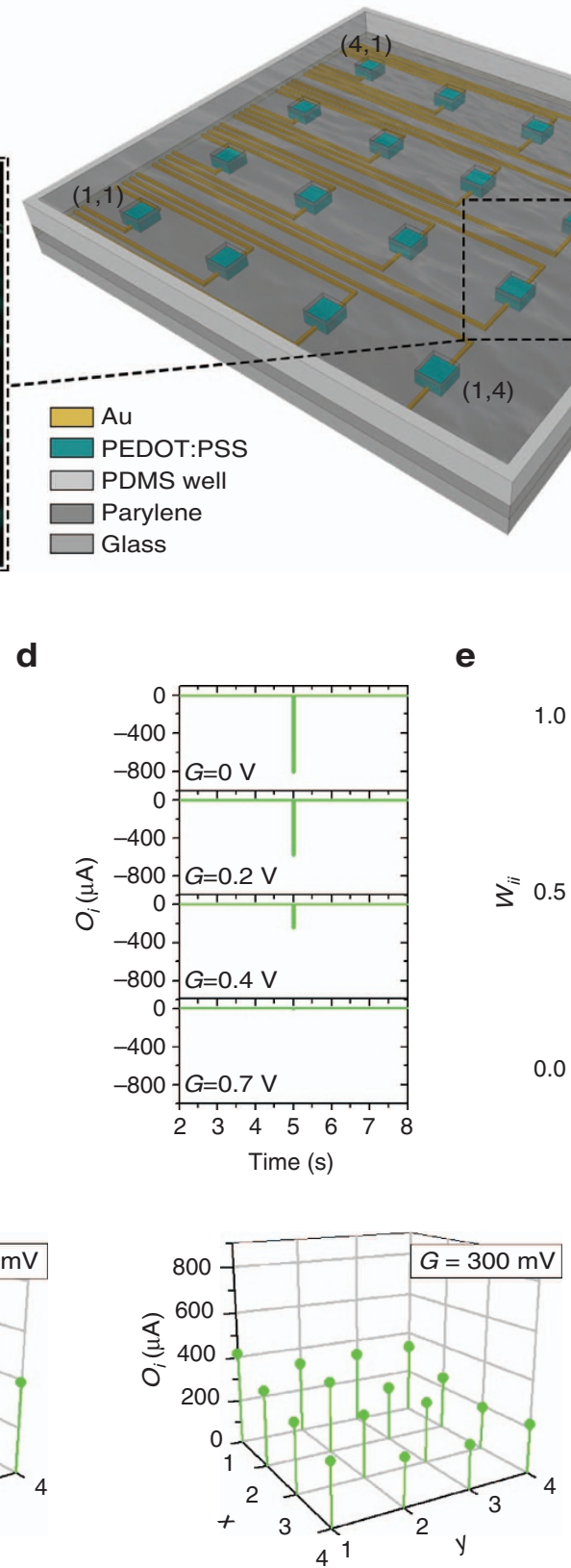

b

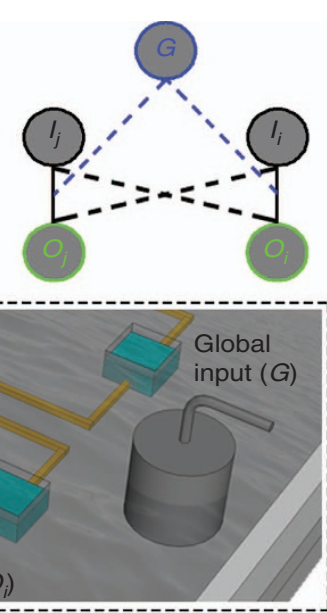

e

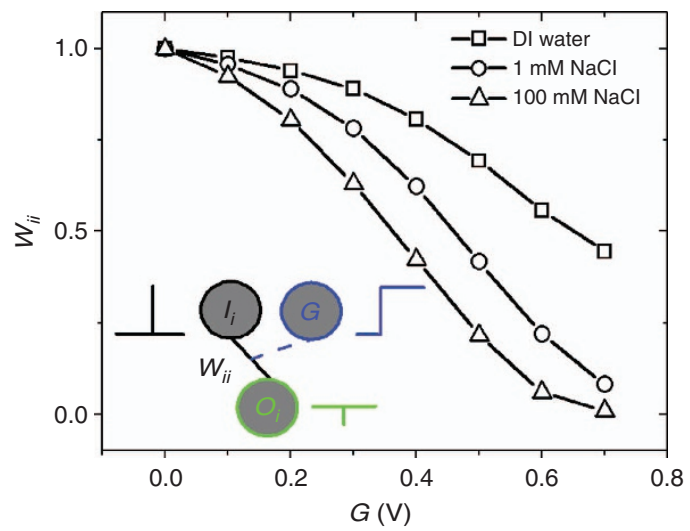

Figure 1 | Configuration of the array and principle of global input. (a) Schematic of the configuration of the array of PEDOT:PSS devices. Each device consists of a PEDOT:PSS channel connected with two Au wires, the local input $l_{i}$ and the output $O_{i}$. The PEDOT:PSS channels are in contact with a common electrolyte in which a $\mathrm{Ag} / \mathrm{AgCl}$ electrode serves as the global input $\mathrm{G}$. The analogy of the device grid and a system of neurons that are immersed in a common electrolyte is also shown. In neural environment global (homeostatic) parameters regulate the overall function of the brain. (b) Representation of the physical (straight line) and soft connections (dash line) that are established in an array of two devices that are immersed in a common electrolyte. (c) Combinations of local and global inputs ( $l_{i}$ : pulse of amplitude of $100 \mathrm{mV}$ and width of $50 \mathrm{~ms}$, G: DC voltage $0-700 \mathrm{mV}$ ), and (d) resulting outputs. (e) Connection weight $w_{i i}$ as a function of the global gate voltage $G$ for different electrolyte concentrations (DI water $-100 \mathrm{mM} \mathrm{NaCl}$ ). Inset shows that weight $w_{i i q}$ quantifies the physical connectivity between input $l_{i}$ and output $O_{i}$, and that it is modulated by the global input. (f) Spatial maps showing the variation of outputs $O_{i}$, when voltages pulses (amplitude of $100 \mathrm{mV}$, width of $50 \mathrm{~ms}$ ) were used at each local input $I_{i}$, and various DC values ( 0 , 300 and $700 \mathrm{mV}$ ) were used as a global input. The $\mathrm{Ag} / \mathrm{AgCl}$ electrode was located as shown in (b). The maps show that global input forces a global restriction on every output.

The effect of the global input is also seen in Fig. 1f, which shows the outputs $O_{i}$ of all devices on the array to be massively suppressed as global gate voltage increases. It should be noted that the variation of $O_{i}$ (Fig. 1f) is attributed to the resistance of the metal connections, which are longer for the devices $(i, 1)$ than $(i, 4)$. Moreover, the influence of the global gate is modulated by the ion concentration in the electrolyte. As seen in Fig. 1e, when the electrolyte concentration is decreased, the influence of the global gate is becoming weaker. Indeed, the output $O_{i}$ is only partially suppressed by the global gate when DI water is used. Detailed gate voltage versus electrolyte concentration spatial mappings of the local output $O_{i}$, presented in Supplementary Fig. 1 (refer also to Supplementary Note 1), show this to happen in all devices of the array. Both gate electrode and electrolyte 
concentration force global restrictions to the output of every device, emulating in this way homeoplasticity phenomena. This global restriction behaviour is practically independent on the exact location of the gate (see also Supplementary Fig. 2 and Supplementary Note 2).

These effects can be understood using the standard model for OECTs, according to which cations are injected from the electrolyte to the channel under the combined influence of the voltage applied at the global and local inputs and decrease the conductivity of the PEDOT:PSS film. This phenomenon can be modelled using an equivalent circuit ${ }^{24,35}$. In the simplest form, this circuit consists of a resistor, representing ionic transport in the electrolyte, in series with a capacitor, representing the charging of PEDOT:PSS with ions. The resistor increases with decreasing ion concentration in the electrolyte, and this increases the RC time and decreases the efficiency of the global gate. Moreover, the influence of various electrolytes on the operation of PEDOT:PSS based devices has also been studied at past ${ }^{36,37}$.
Synchronization functions. The global input can be used to impose synchronization of $\mathrm{I} / \mathrm{O}$ transmission. As shown in (Fig. 2a,b), a voltage pulse at the global input temporarily shuts down transmission of the local input of the device at coordinates $(1,1)$. This behaviour can be utilized to synchronize globally the I/O transmission of an array of devices. For example, in Fig. 2c, a train of voltage pulses at the local input is transmitted to the output, unless a voltage pulse is applied at the global input at the same time (which happens at $t \sim 10 \mathrm{~s}$ ). Figure $2 \mathrm{~d}-\mathrm{f}$ shows that an inverted pulse at the global input has the opposite effect, namely enabling local I/O transmission during its presence. In Fig. $2 c, f$, a train of pulses is used as local input with amplitude of $220 \mathrm{mV}$, offset of $80 \mathrm{mV}$, width of $10 \mathrm{~ms}$, in order to study the $\mathrm{I} / \mathrm{O}$ transmission. The same phenomenon can be used to implement a global clock. As shown in Fig. 3a,b an oscillatory voltage at the global input modulates a train of voltage pulses at the local input. Indeed, the connection weight $w_{i i}$ is modulated within the entire range (from 0 to 1 ), and in a purely analogue manner.

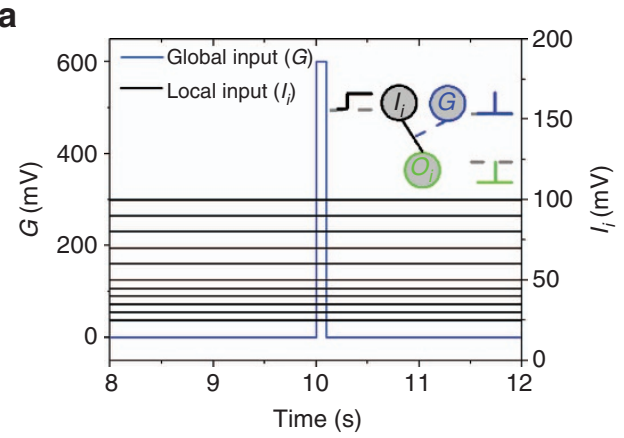

b

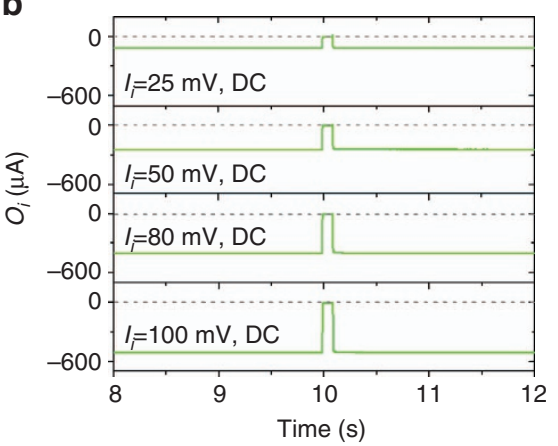

C

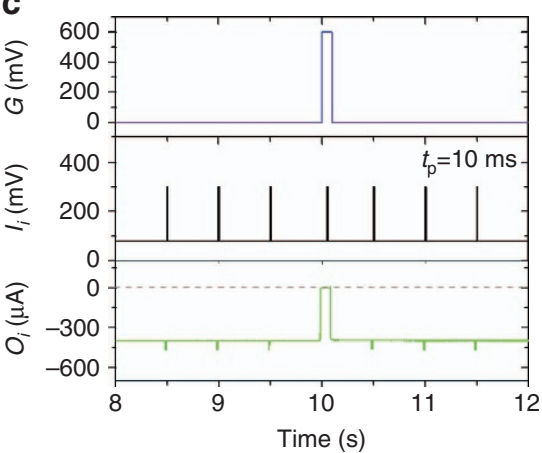

d

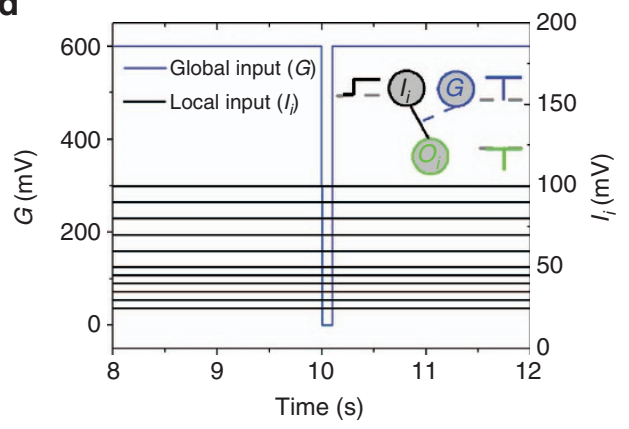

e

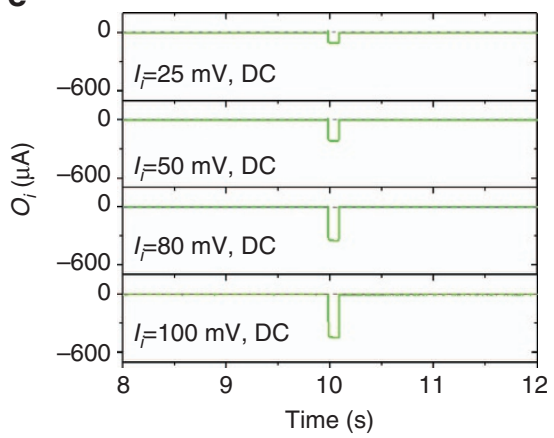

f

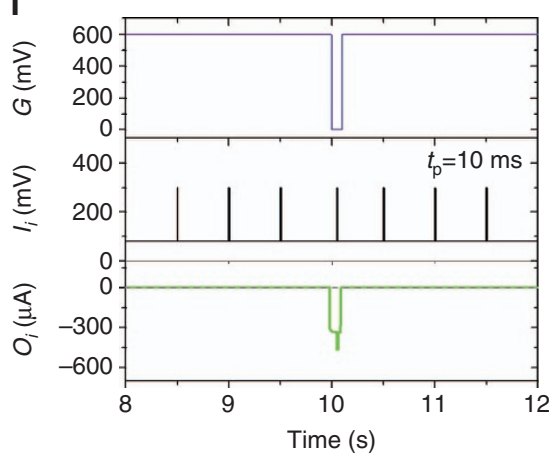

Figure 2 | Synchronization of I/O transmission with a global input. (a) Combinations of local and global inputs ( $I_{i}$ : DC voltage 25-100 mV, G: pulse of amplitude of $600 \mathrm{mV}$ and width of $50 \mathrm{~ms}$ ), and (b) resulting outputs, showing that $l_{i} / O_{i}$ transmission shuts down when a voltage pulse is applied at the global input. (c) The same pulse shuts down transmission of voltage pulses (amplitude of $220 \mathrm{mV}$, offset of $80 \mathrm{mV}$, width of $10 \mathrm{~ms}$ ) applied at the local input. (d) Combinations of local and global inputs, now with an inverted pulse as the global input, and (e) resulting outputs showing that transmission is enabled during the inverted pulse. (f) The same pulse now enables transmission of local input. 
a

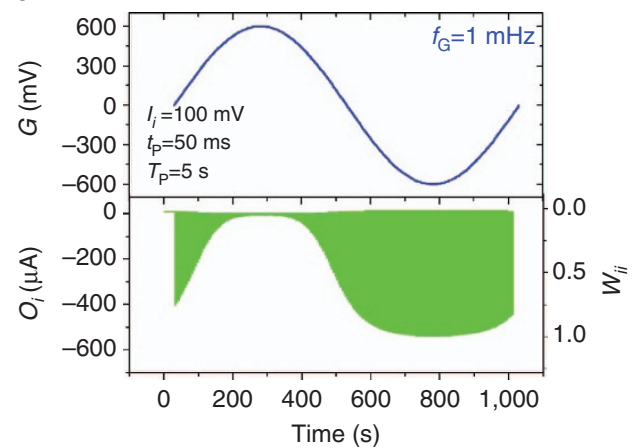

C

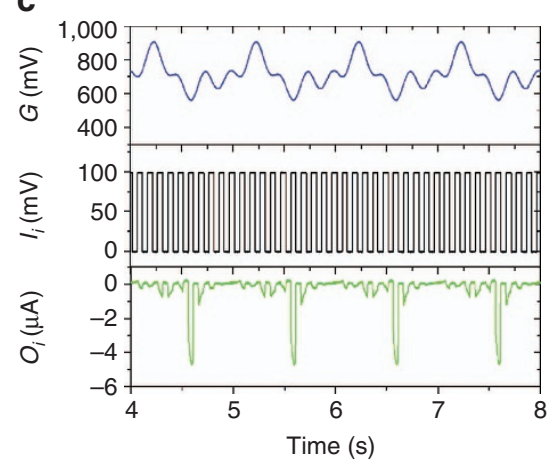

b

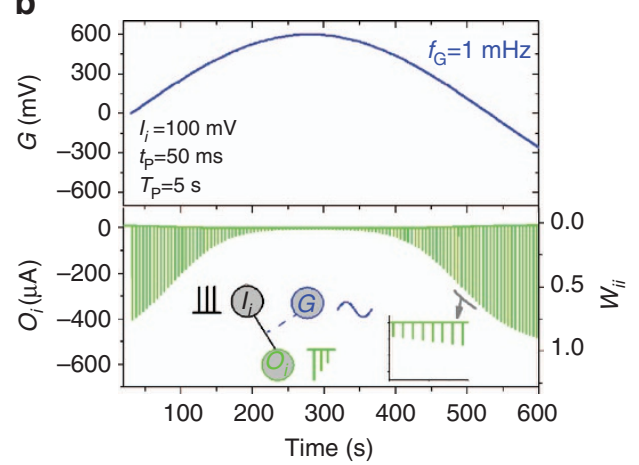

d

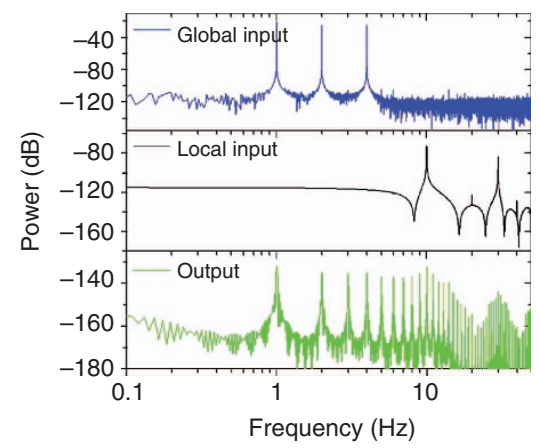

Figure 3 | Global input as a global clock. (a,b) Periodic modulation of the output caused by a train of voltage pulses (amplitude of $100 \mathrm{mV}$, width of $50 \mathrm{~ms}$, period of $5 \mathrm{~s}$ ) at a local input, and a sine wave (amplitude of $\pm 600 \mathrm{mV}$, frequency of $1 \mathrm{mHz}$ ) at the global input, shown here for a full period (a) and for the positive half of the cycle (b). (c) Global input with higher harmonics (amplitude modulation of a sine wave of frequency of $1 \mathrm{~Hz}$, amplitude of $180 \mathrm{mV}$ and offset of $700 \mathrm{mV}$, with a second sine wave of frequency of $3 \mathrm{~Hz}, \mathrm{AM}$ depth of $120 \%$ ), and a train of voltage pulses (amplitude of $100 \mathrm{mV}$, width of $50 \mathrm{~ms}$, period of $100 \mathrm{~ms}$ ) at local input results to an output that contains predominantly the low-frequency components of the global input. (d) Power spectra of $G, I_{i}$ and $O_{i}$.

This happens simultaneously for all the devices in the array (Supplementary Fig. 3, Supplementary Note 3), hence the oscillation acts as a global clock, allowing transmission of local input signals only during certain periods of time.

Synchronization is also achieved when a voltage oscillation containing higher harmonics is used as the global input. In Fig. 3c the global input imposes periods of time during which $\mathrm{I} / \mathrm{O}$ transmission suppressed, and this permits the synchronization of a local input signal with a global oscillation at its basic harmonic of $1 \mathrm{~Hz}$. More specifically, a global oscillation chosen with a positive offset is chosen (in the range $500-900 \mathrm{mV}$ ), in order to operate the device in the sub-threshold regime (that is, $G>500 \mathrm{mV}$, or the 'OFF' state) during the majority of the global oscillation, and only during the basic harmonic of the AC signal (that is, $1 \mathrm{~Hz}$ ) the device is operated close to the threshold regime $(G \sim 500 \mathrm{mV})$. This permits the implementation of synchronization functions. Power spectra of the input and output signals are shown in Fig. 3d. The spectral density of the output signal displays the basic harmonics of the global oscillation, which are the 1, 2 and $4 \mathrm{~Hz}$ (see also Supplementary Note 4). The spectral density also shows a range of higher frequencies along with the basic harmonics, due to the need of a whole range of harmonics for the construction of the complex structure of the current output $O_{i}$ of Fig. 3c. Moreover, when a steeper oscillation is used as a global signal, this results in higher output current $O_{i}$ (see also Supplementary Fig. 4). The low-frequency harmonics of the global input are transmitted to the output. Therefore the global input plays the role of a synchronization function similar to that in biological systems, where low-frequency global neural oscillations provide discrete activation windows for the neural response 38,39 . Notably, the behaviour of Fig. 3c,d also resembles the phenomenon of neural entrainment, which is related to the synchronization of the activity of neural ensembles with periodic external stimuli (that is, acoustic or optical) ${ }^{40,41}$.

Soft connections thought the electrolyte. In addition to providing a global input, the electrolyte also introduces soft connections between the inputs and outputs of different devices and enables their communication. As described in Supplementary Note 5, effectively each device acts as a gate for all the other devices (see also Supplementary Fig. 5). The concept is demonstrated in Fig. $4 \mathrm{a}$, where the input $I_{j}$ of the $j$ th device also acts as an input to an adjacent $i$ th device, with the coupling between the $I_{j}$ input and the $O_{i}$ output characterized by the connection weight $w_{j i}$. Here the term connection weight is used to express the correlation of a corresponding lateral output in respect to a physical output. A spatial map of the output $O_{i}$ of the device at coordinates $(1,1)$, that results from applying an input pulse at every local input $I_{j}$, of devices of the array is depicted at Fig. $4 \mathrm{~b}$. The results show that a local input has an effect on all devices of the array. The spatial mapping of the connection weight $w_{j i}$ (defined here as $O_{i} / O_{\mathrm{MAX}, i}$, where $O_{\mathrm{MAX}, i}$ is the output that results from each corresponding $i$ th local input $I_{i}$ ) is depicted in Fig. 4c. When compared to the physical connection weight $w_{i i}$ (see also Fig. 1d), the soft connection weight $w_{j i}$ is weaker. For example, a soft connection between a jth local input and an ith output, creates an output $O_{i}$ that is $\sim 5 \%$ of the output elicited by the corresponding local input. Figure $4 \mathrm{c}-\mathrm{e}$ show the spatial mappings of $w_{j i}$. Similarly to hard connection weights, the soft connection weight $w_{j i}$ 
a

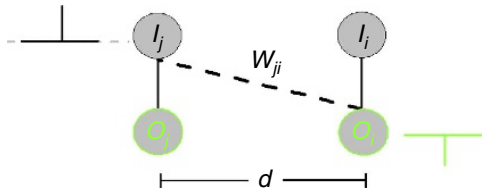

C

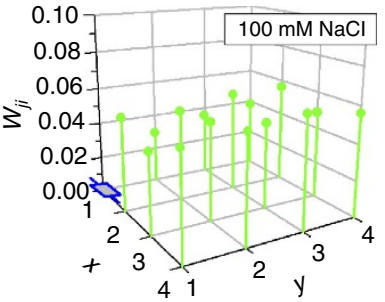

d b
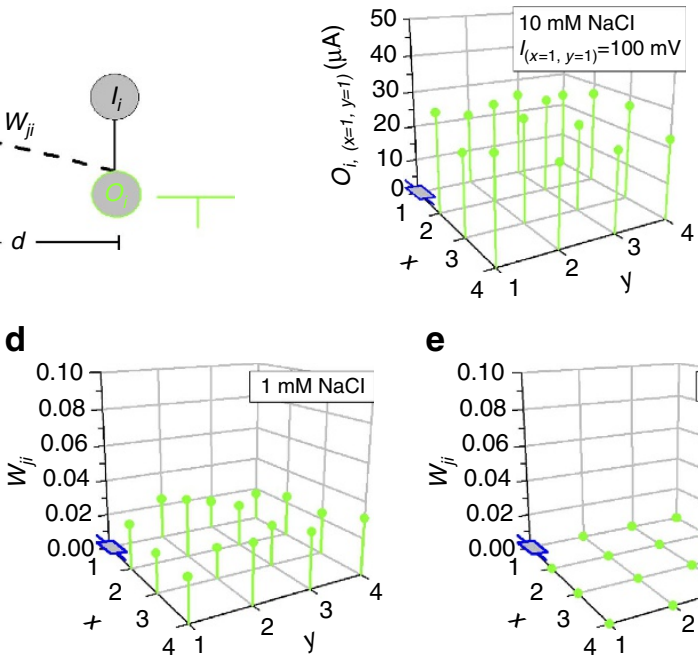

e

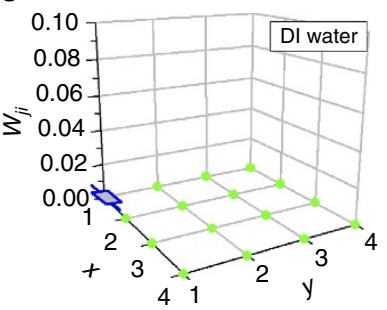

Figure 4 | Lateral communication of devices through soft electrolyte connections. (a) Schematic of lateral communication and definition of the soft connection weight $w_{j i}$. (b) Spatial map of the output $O_{i,(x=1, y=1)}$ of the device at coordinates $(1,1)$ when a pulse is applied at local input $I_{j}(x \neq 1, y \neq 1)$ (amplitude of $100 \mathrm{mV}$, width of $50 \mathrm{~ms}$ ) of devices at coordinates $x \neq 1, y \neq 1$. The distance $d$ between the devices is $5 \mathrm{~mm}$. (c-e) Spatial mapping of the connection weight $w_{j i}$ (defined as $\left.O_{i} / O_{M A X}, i\right)$ for different electrolyte concentrations (100 mM, $1 \mathrm{mM}$, DI water).

a

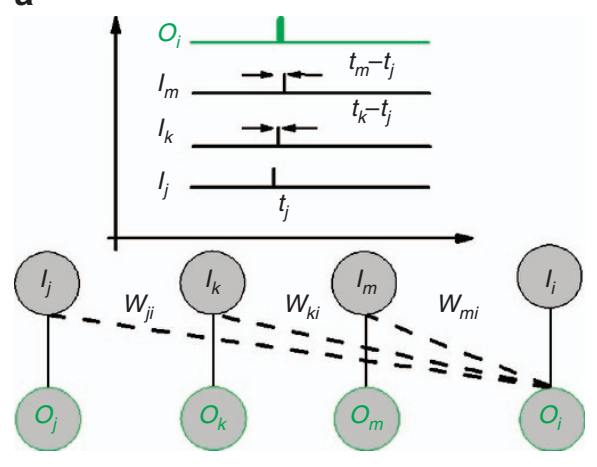

b

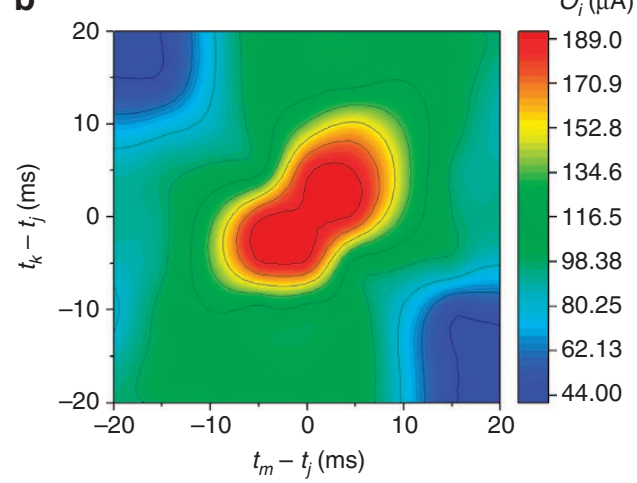

Figure 5 | Coincidence detection using soft connections between devices. (a) Schematic of the measurement protocol. A voltage pulse (amplitude of $0.5 \mathrm{~V}$, width of $10 \mathrm{~ms}$ ) is applied at three local inputs $l_{j}, I_{k}, I_{m}$ with variable time intervals $t_{k}-t_{j}$ and $t_{m}-t_{j}$ with respect to a reference time $t_{j}$ of the input $l_{j}$. The output $O_{i}$ of the ith device is measured for different timing conditions. (b) Time correlation map of the output $O_{i}$ showing coincidence detection.

also depends on the electrolyte concentration. As the electrolyte concentration decreases, the weight $w_{j i}$ decreases (from $\sim 5 \%$ $O_{\mathrm{MAX}, i}$ for $100 \mathrm{mM}$ to $\sim 2 \% O_{\mathrm{MAX}, i}$ for $1 \mathrm{mM}$ ), and in the case of DI water, lateral connectivity between individual devices is completely suppressed $\left(\mathrm{w}_{\mathrm{ji}} \sim 0 \% \mathrm{O}_{\mathrm{MAX}, i}\right)$.

Coincidence detection. The availability of soft lateral connections between devices endowed by the electrolyte can be leveraged to realize multi-terminal functionalities from individual devices without complex wiring. This behaviour has important implications in neuromorphic computing because in this way, complex summations from multiple inputs can be performed in another output through the electrolyte continuum without predefined physical connections. In biological neural networks the parallelism originates from the massive interconnections between the neurons through the synapses. The concept of interconnectivity is very important in the actual neural environment and is a key feature for reliable computing with unreliable neurons, in which there is a redundancy of interconnections. Figure 5a,b demonstrates the way that time correlated signals from various spatially distributed local inputs are superimposed in a separate output $\left(O_{i}\right)$. Here, voltage pulses are applied at three local inputs $\left(I_{j}, I_{k}, I_{m}\right)$ with variable time intervals $t_{k}-t_{j}$ (for the $I_{k}$ input) and $t_{m}-t_{j}$ (for the $I_{m}$ input) with respect to a reference time $t_{j}$ of the $I_{j}$ input (Fig. 5a), and the output $O_{i}$ of the $i$ th device is measured (refer also to Supplementary Fig. 6 and Supplementary Note 6). Following the above mentioned measurement protocol, a time correlation map of the output $O_{i}$ is constructed and depicted in Fig. 5b. The map shows that a maximum output $O_{i}$ results when all inputs $I_{j}, I_{k}, I_{m}$, are time synchronized (that is, when $t_{m}-t_{j}$, $\left.t_{k}-t_{j} \sim 0\right)$. Secondary maximums of the $O_{i}$ also appear on the map, which are attributed to the synchronization of just two inputs. The overall output response $O_{\mathrm{i}}$ is slightly super-linear summation of the individual local inputs (see also Supplementary Fig. 7 and Supplementary Note 7). The occurrence of spatially distributed, multiple inputs is encoded through soft connections in the response of $O_{i}$, a behaviour that resembles coincidence detection in biological neural networks. Coincidence detection neurons play important role in neural signalling, especially in the visual $^{42}$, and auditory system ${ }^{43,44}$. In the binaural cochlear for example, coincidence detection is regarded as a mechanism for 
conversion of acoustic to spatial codes and it is thus used in the auditory system for spatial localization. The fact that such multiterminal functionalities are implemented by taking advantage of soft connections in a device array with minimal hard wiring highlights the utility of electrolyte as connectivity media in neuromorphic systems.

\section{Discussion}

Previous papers on neuromorphic devices explored the role of electrolytes in connecting one $e^{10,11}$, or multiple inputs to a single device ${ }^{12,45,46}$. In this work, we use a common electrolyte to connect and control a whole array of devices. The PEDOT:PSS channels represent hardwired connections between inputs and outputs, and their connection strengths can be globally modulated by the applied voltage and by the ion concentration in the electrolyte. This enables slowly-varying AC voltage signals at the electrolyte to be used as global clocks that regulate periodically the I/O transmission. Other global clocks, such as periodic changes in ionic concentration, are also expected to regulate the $\mathrm{I} / \mathrm{O}$ transmission and connection weights and this might lead to applications in biosensing. Most importantly, electrolyte gating is shown to exhibit global inhibitory characteristics and is used to massively suppress connectivity in the entire array. This is a key feature for neuromorphic devices, in order to emulate homeostatic plasticity phenomena of the neural environment ${ }^{29,31}$.

In this work, we use volatile PEDOT:PSS devices in order to provide the simplest demonstration of global gating. Other PEDOT-based derivatives that exhibit memory phenomena ${ }^{11,47}$, can be used to introduce long-term-memory phenomena and lead to more complex network behaviours. Moreover, the performance of the architecture discussed here can be tuned through the use of anisotropic ionic conductors ${ }^{48}$ as global electrolytes, which are expected to define spatial- or directionaldependent soft connection weights. Furthermore, ion-selective conjugated polymers as active materials ${ }^{49}$, can be leveraged to implement unique features such as local processing of ionic signals from various ionic species. Finally, the phenomena described here, such as the influence of applied voltage and ion concentration in the electrolyte on conductivity of PEDOT:PSS, have been studied in conjunction with the development of OECTs, and quantitative models exist to describe them. The global gate/electrolyte/PEDOT:PSS stack, for example, is identical to the ionic part of the circuit of an OECT, which is modelled using simple equivalent circuits. The availability of such models allows the fine tuning of the response of neuromorphic devices in order to for example, access specific timescales required in particular applications.

\footnotetext{
Methods

Device fabrication. The devices were fabricated using standard microfabrication techniques. As substrates, $26 \times 76 \mathrm{~mm}$ glass slides were used. The contact lines were defined by evaporating a $10 \mathrm{~nm} \mathrm{Cr}$ and a $100 \mathrm{~nm}$ Au layer on top of prepatterned photoresist and a subsequent soaking in an acetone/isopropanol bath to remove the excess material. The $\mathrm{Cr}$ layer is needed to enhance surface adhesion between glass and Au. To protect the contact lines from the electrolyte, two parylene $\mathrm{C}$ layers were deposited on top, with $2 \mu \mathrm{m}$ thickness each. The first was deposited on a surface treated with silane to enhance adhesion with the substrate and a thin $2 \%$ soap solution layer was spin-coated before the second deposition. This allows to peel-off the upper layer, thereby defining the device active area with nominal dimensions of $50 \mu \mathrm{m} \times 50 \mu \mathrm{m}$ (length $\times$ width, $L \times W$ ). The polymer used in this communication was PEDOT:PSS [Clevios PH 1,000 from Heraeus Holding $\mathrm{GmbH}$, with $5 \mathrm{wt} \%$ ethylene glycol, $0.1 \mathrm{wt} \%$ dodecyl benzene sulf acid and $1 \mathrm{wt} \%$ of (3-glycidyloxypropyl) trimethoxysilane)]. Three subsequent spin-coating steps with varying rotation speeds of 1,500, 650 and 650 r.p.m., respectively, yielded a film thickness of $\sim 500 \mathrm{~nm}$. A second photolithographic step was used to pattern these features. PEDOT:PSS spin-coating was followed by a hard-bake step at $140^{\circ} \mathrm{C}$ for $60 \mathrm{~min}$. Using the above mentioned process, an array of $4 \times 4$ devices was fabri-
} cated with centre-to-centre distance $d=5 \mathrm{~mm}$. For every input and output terminal, metal pads that extend outside the electrolyte region and were not covered with parylene were used.

Device characterization. The array of $4 \times 4$ devices was gated with a global $\mathrm{Ag} / \mathrm{Cl}$ gate electrode and aqueous $\mathrm{NaCl}$ electrolyte $(1-100 \mathrm{mM})$ in deionized water. Measurements were recorded after cycling the every device (repetitive $0.3 /-0.3 \mathrm{~V}$ cycles at the gate for $2 \mathrm{~s}$ each) in order to obtain reproducible behaviour (endurance measurements are presented in Supplementary Fig. 8 and Supplementary Note 8). The current of the $O$ terminal was measured using a National Instrument PXIe1062Q system. The devices were biased with a PXIe-4145 source measure unit that was simultaneously recording the current at the output with a sampling rate of $1 \mathrm{kHz}$. Voltages for the local and global inputs were generated by a National Instruments USB-6259. Both $G, I$ voltages and $O$ current measurements were internally triggered by the PXIe system. For the measurement of soft connectivity, a bias of $-50 \mathrm{mV}$ was applied to the device whose output was measured, and the global gate electrode was disconnected. The input signal containing higher harmonics was generated with a Keithley 3,390 Arbitrary Function Generator. The acquisition system was controlled by custom-made LabVIEW software. Fast Fourier Transform was performed using OriginPro software.

Data availability. The data that support the findings of this study are available from the corresponding authors upon request.

\section{References}

1. Service, R. F. The brain chip. Science 345, 614-616 (2014).

2. Backus, J. Can programming be liberated from the von Neumann style?: a functional style and its algebra of programs. Commun. ACM 21, 613-641 (1978).

3. Mead, C. Neuromorphic electronic systems. Proc. IEEE 78, 1629-1636 (1990).

4. Merolla, P. A. et al. A million spiking-neuron integrated circuit with a scalable communication network and interface. Science 345, 668-673 (2014).

5. Duygu, K., Shimeng, Y. \& Wong, H. S. P. Synaptic electronics: materials, devices and applications. Nanotechnology 24, 382001 (2013).

6. Jo, S. H. et al. Nanoscale memristor device as synapse in neuromorphic systems. Nano Lett. 10, 1297-1301 (2010).

7. Tuma, T., Pantazi, A., Le Gallo, M., Sebastian, A. \& Eleftheriou, E. Stochastic phase-change neurons. Nat. Nanotechnol. 11, 693-699 (2016).

8. Locatelli, N., Cros, V. \& Grollier, J. Spin-torque building blocks. Nat. Mater. 13, 11-20 (2014).

9. Chanthbouala, A. et al. A ferroelectric memristor. Nat. Mater. 11, 860-864 (2012).

10. Gkoupidenis, P., Schaefer, N., Garlan, B. \& Malliaras, G. G. Neuromorphic functions in PEDOT:PSS organic electrochemical transistors. Adv. Mater. 27, 7176-7180 (2015).

11. Gkoupidenis, P., Schaefer, N., Strakosas, X., Fairfield, J. A. \& Malliaras, G. G. Synaptic plasticity functions in an organic electrochemical transistor. Appl. Phys. Lett. 107, 263302 (2015).

12. Gkoupidenis, P., Koutsouras, D. A., Lonjaret, T., Fairfield, J. A. \& Malliaras, G. G. Orientation selectivity in a multi-gated organic electrochemical transistor. Sci. Rep. 6, 27007 (2016).

13. Tarabella, G. et al. A hybrid living/organic electrochemical transistor based on the physarum polycephalum cell endowed with both sensing and memristive properties. Chem. Sci. 6, 2859-2868 (2015).

14. Romeo, A. et al. A bio-inspired memory device based on interfacing Physarum polycephalum with an organic semiconductor. APL Mater. 3, 014909 (2015).

15. Alibart, F. et al. An organic nanoparticle transistor behaving as a biological spiking synapse. Adv. Funct. Mater. 20, 330-337 (2010).

16. Xu, W., Min, S.-Y., Hwang, H. \& Lee, T.-W. Organic core-sheath nanowire artificial synapses with femtojoule energy consumption. Sci. Adv. 2, e1501326 (2016).

17. Berggren, M. \& Richter-Dahlfors, A. Organic bioelectronics. Adv. Mater. 19, 3201-3213 (2007).

18. Khodagholy, D. et al. In vivo recordings of brain activity using organic transistors. Nat. Commun. 4, 1575 (2013).

19. Strakosas, X., Bongo, M. \& Owens, R. M. The organic electrochemical sistor for biological applications. J. Appl. Polym. Sci. 132, 41735-41748 (2015).

20. Simon, D. T. et al. Organic electronics for precise delivery of neurotransmitters to modulate mammalian sensory function. Nat. Mater. 8, 742-746 (2009).

21. Hemmatian, Z. et al. Taking electrons out of bioelectronics: bioprotonic memories, transistors, and enzyme logic. J. Mater. Chem. C 3, 6407-6412 (2015).

22. Stavrinidou, E. et al. Electronic plants. Sci. Adv. 1, e1501136 (2015).

23. White, H. S., Kittlesen, G. P. \& Wrighton, M. S. Chemical derivatization of an array of three gold microelectrodes with polypyrrole: fabrication of a moleculebased transistor. J. Am. Chem. Soc. 106, 5375-5377 (1984).

24. Bernards, D. A. \& Malliaras, G. G. Steady-State and transient behavior of organic electrochemical transistors. Adv. Funct. Mater. 17, 3538-3544 (2007). 
25. Gkoupidenis, P., Rezaei-Mazinani, S., Proctor, C. M., Ismailova, E. \& Malliaras, G. G. Orientation selectivity with organic photodetectors and an organic electrochemical transistor. AIP Adv. 6, 111307 (2016).

26. Wan, C., Wu, G., Guo, L., Zhu, L. \& Wan, Q. Artificial synaptic arrays intercoupled by nanogranular proton conductors for building neuromorphic systems. Preprint at https://arxiv.org/abs/1301.2052 (2013).

27. Machens, C. K. Building the human brain. Science 338, 1156-1157 (2012).

28. Kandel, E. R., Schwartz, J. H. \& Jessell, T. M. Principles of Neural Science (McGraw-Hill, Health Professions Division, 2000).

29. Turrigiano, G. G. \& Nelson, S. B. Homeostatic plasticity in the developing nervous system. Nat. Rev. Neurosci. 5, 97-107 (2004).

30. Queenan, B. N., Lee, K. J. \& Pak, D. T. S. Wherefore art thou, homeo(stasis)? functional diversity in homeostatic synaptic plasticity. Neural Plast. 2012, 12 (2012).

31. Abbott, L. F. \& Nelson, S. B. Synaptic plasticity: taming the beast. Nat. Neurosci. 3, 1178-1183 (2000).

32. Miller, K. D. \& MacKay, D. J. C. The role of constraints in hebbian learning Neural Comput. 6, 100-126 (1994).

33. Miller, K. D. Synaptic economics: competition and cooperation in synaptic plasticity. Neuron 17, 371-374 (1996).

34. Royer, S. \& Pare, D. Conservation of total synaptic weight through balanced synaptic depression and potentiation. Nature 422, 518-522 (2003).

35. Rivnay, J. et al. High-performance transistors for bioelectronics through tuning of channel thickness. Sci. Adv. 1, e1400251 (2015)

36. Kumar, P. et al. Effect of channel thickness, electrolyte ions, and dissolved oxygen on the performance of organic electrochemical transistors. Appl. Phys. Lett. 107, 053303 (2015).

37. Khodagholy, D. et al. High transconductance organic electrochemical transistors. Nat. Commun. 4, 2133 (2013).

38. Buzsáki, G. \& Draguhn, A. Neuronal oscillations in cortical networks. Science 304, 1926-1929 (2004).

39. Buzsáki, G. \& Watson, B. O. Brain rhythms and neural syntax: implications for efficient coding of cognitive content and neuropsychiatric disease. Dialogues Clin. Neurosci. 14, 345-367 (2012).

40. Will, U. \& Berg, E. Brain wave synchronization and entrainment to periodic acoustic stimuli. Neurosci. Lett. 424, 55-60 (2007)

41. Nozaradan, S., Peretz, I. \& Keller, P. E. Individual differences in rhythmic cortical entrainment correlate with predictive behavior in sensorimotor synchronization. Sci. Rep. 6, 20612 (2016).

42. König, P., Engel, A. K. \& Singer, W. Integrator or coincidence detector? The role of the cortical neuron revisited. Trends Neurosci. 19, 130-137 (1996).

43. Joris, P. X., Smith, P. H. \& Yin, T. C. T. Coincidence detection in the auditory system. Neuron 21, 1235-1238 (1998).

44. Agmon-Snir, H., Carr, C. E. \& Rinzel, J. The role of dendrites in auditory coincidence detection. Nature 393, 268-272 (1998).

45. Wan, C. J. et al. Proton-conducting graphene oxide-coupled neuron transistors for brain-inspired cognitive systems. Adv. Mater. 28, 3557-3563 (2016).

46. Zhu, L. Q., Wan, C. J., Guo, L. Q., Shi, Y. \& Wan, Q. Artificial synapse network on inorganic proton conductor for neuromorphic systems. Nat. Commun. 5, 3158 (2014)
47. Winther-Jensen, B., Kolodziejczyk, B. \& Winther-Jensen, O. New one-pot poly(3,4-ethylenedioxythiophene): poly(tetrahydrofuran) memory material for facile fabrication of memory organic electrochemical transistors. APL Mater. 3, 014903 (2015).

48. Kishimoto, K. et al. Nanostructured anisotropic ion-conductive films. J. Am. Chem. Soc. 125, 3196-3197 (2003).

49. Giovannitti, A. et al. Sodium and potassium ion selective conjugated polymers for optical ion detection in solution and solid state. Adv. Funct. Mater. 26, 514-523 (2016).

\section{Acknowledgements}

Financial support from the Marie Curie ITN OLIMPIA is acknowledged. The authors wish to thank Themis Prodromakis and Alexander Serb from the University of Southampton, and Marcel Brändlein from the Department of Bioelectronics for fruitful discussions. The authors also acknowledge Thomas Lonjaret from the Department of Bioelectronics for the development of the acquisition software.

\section{Author contributions}

G.G.M. and P.G. conceived the experiments. D.A.K. fabricated the devices. P.G. performed the electrical characterization of the devices, and analysed the data. G.G.M. and P.G. wrote the manuscript, and all authors participated in manuscript input and editing.

\section{Additional information}

Supplementary Information accompanies this paper at http://www.nature.com/ naturecommunications

Competing interests: The authors declare no competing financial interests.

Reprints and permission information is available online at http://npg.nature.com/ reprintsandpermissions/

How to cite this article: Gkoupidenis, P. et al. Neuromorphic device architectures with global connectivity through electrolyte gating. Nat. Commun. 8, 15448 doi: $10.1038 /$ ncomms15448 (2017).

Publisher's note: Springer Nature remains neutral with regard to jurisdictional claims in published maps and institutional affiliations.

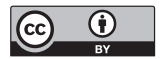

This work is licensed under a Creative Commons Attribution 4.0 International License. The images or other third party material in this article are included in the article's Creative Commons license, unless indicated otherwise in the credit line; if the material is not included under the Creative Commons license, users will need to obtain permission from the license holder to reproduce the material. To view a copy of this license, visit http://creativecommons.org/licenses/by/4.0/

(C) The Author(s) 2017 